\title{
Influence of Hartmann Number on Convective Flow of Maxwell Fluid between Two Hot Parallel Plates through Porous Medium Subject to Arbitrary Shear Stress at the Boundary
}

\author{
Adnan Ahmad1, Zaib Un Nisa2, Mudassar Nazar ${ }^{1,3 *}$, Muhammad Imran Asjad4, Mushtaq Ahmad1 \\ ${ }^{1}$ Centre for Advanced Studies in Pure and Applied Mathematics, Bahauddin Zakariya University, Multan, Pakistan \\ ${ }^{2}$ Department of Mathematics, University of Education Lahore, Multan Campus, Multan, Pakistan \\ ${ }^{3}$ School of Mathematical Sciences, University of Science and Technology of China, Hefei, China \\ ${ }^{4}$ Department of Mathematics, University of Management and Technology, Lahore, Pakistan \\ Email: mudassar_6@yahoo.com
}

How to cite this paper: Ahmad, A., Nisa, Z.U., Nazar, M., Asjad, M.I. and Ahmad, M. (2022) Influence of Hartmann Number on Convective Flow of Maxwell Fluid between Two Hot Parallel Plates through Porous Medium Subject to Arbitrary Shear Stress at the Boundary. Journal of Applied Mathematics and Physics, 10, 160-171.

https://doi.org/10.4236/jamp.2022.101012

Received: November 26, 2021

Accepted: January 18, 2022

Published: January 21, 2022

Copyright $\odot 2022$ by author(s) and Scientific Research Publishing Inc. This work is licensed under the Creative Commons Attribution International License (CC BY 4.0).

http://creativecommons.org/licenses/by/4.0/ (c) (i) Open Access

\begin{abstract}
Natural convection flow of unsteady Maxwell fluid with the effects of constant magnetic force in the course of a porous media is investigated in this research work. Fluid motion between a channel of parallel plates is tempted by time dependent shear stress applied on one plate. The governing partial differential equations of a model under consideration are transformed into ordinary differential equations by Laplace transform method and then solved for temperature and velocity fields. The obtained results for temperature fields are expressed in terms of complementary error function. The influences of involved parameters likes Hartmann number, Grashf number, Prandlt number and porosity parameter, on temperature and velocity profiles are shown graphically. There is no such result regarding Maxwell fluid in the existing literature.
\end{abstract}

\section{Keywords}

Natural Convection, Maxwell Fluid, Hot Parallel Plates, MHD, Porous Media, Shear Stress

\section{Introduction}

Free convection flow of Maxwell fluid subject to the magnetic field (MHD) through a porous medium has remarkable applications in engineering industry. A porous medium is formed by voids and solid matrix interconnection. Such 
media allow fluids to flow and have various useful applications in everyday life and manufacturing sciences [1] [2] [3]. Furthermore, an electrically conducting fluid motion in a magnetic field is known as magnetohydrodynamics. Magnetohydrodynamics (MHD) has significant effects on fluid flow and can be observed in numerous natural and artificial flows. Fluids flow under the influence of magnetic force has several uses in field of science industry, such as: thermal and nuclear power generation, pumping the liquids and gases, manufacturing of electric pump and many others [4] [5]. In the preceding few years, models of rate type MHD fluids embedded in permeable medium have been studied for engineering and biological applications by many mathematicians and scientists [6]-[11].

Flow of non-Newtonian fluids has not only basic mathematical concern, but it is also appeared in several useful and applicable process, e.g. drilling, blood flow, cooling and heating process, artificial surfing etc. [12]-[25]. Sultan et al. [26] have made the clarification for flow of generalized Burgers fluid through channel of parallel walls subject to rectified sine pulses stress with the existence of uniform magnetic field. Khan et al. [27] analyzed free convection flow of viscous fluid for mass transfer on an infinite inclined plane with MHD. Riaz et al. [28] and Akhtar et al. [29] discussed the motion of generalized Maxwell fluid and obtained the exact solutions.

In the present study, the flow of unsteady MHD Maxwell fluid flow embedded porous medium in channel of two side plates, is considered. The motion of one plate is generated by unsteady shear stress while the other is fixed. The Laplace transform is initially applied on the governing equation for the developed model. Then, the Stehfest's and Tzou's algorithms are applied to obtain the inversion of transformed results. The obtained results for solutions of problem are also presented graphically and the effects of potent parameters are explained by some graphs. According to our best knowledge, this piece of research work is still not considered in the existing literature.

\section{Statement of Problem}

We reckon a natural convection flow of Maxwell fluid of constant density in a porous medium in the presence of magnetic field through a couple of hot side plates situated in rectangular coordinates system in such a way that their planes lies in xz-plane and separated by distance d. Initially, the whole system with fluid and its constrains is in the state of equilibrium at a constant thermal condition $T_{0}$. For time $t>0$ the left plat begins to move under the applied time dependent shear stress in its own plane consequently the fluid flow have been induced. The temperature of this plate hoist or falls according to $T_{0}+\left(T_{w}-T_{0}\right) g(t)$ where $g(t)$ is continues function satisfy the $g(0)=0$. A homogeneous magnetic force field of the strength $B_{0}$ normal to the plate surface is applied which is also normal to the flow direction. Here we presume that fluid motion and its temperature are functions of special variable $y$ and time variable $t$, also induced 
magnetic field and convective effects can be neglected. Dissipation due to viscosity and Joule heating in energy momentum equations are negligible. With the standard Boussinesq's approximation, governing equations for incompressible and unsteady fluid flow are as

$$
\begin{gathered}
v \frac{\partial^{2} u(y, t)}{\partial y^{2}}+\left(1+\lambda_{1} \frac{\partial}{\partial t}\right) g \beta\left(T-T_{d}\right)-\left(1+\lambda_{1} \frac{\partial}{\partial t}\right) \frac{\sigma B_{0}^{2}}{\rho} u(y, t)-\frac{\phi v}{k_{1}} u \\
=\left(1+\lambda_{1} \frac{\partial}{\partial t}\right) \frac{\partial u}{\partial t}, \\
k \frac{\partial^{2} T(y, t)}{\partial y^{2}}=\rho c_{p} \frac{\partial T(y, t)}{\partial t},
\end{gathered}
$$

appropriate initial and boundary conditions are

$$
\begin{gathered}
u(y, 0)=0=\frac{\partial u(y, 0)}{\partial t}, T(y, 0)=T_{d}, 0 \leq y \leq d, \\
\left.\left(1+\lambda_{1} \frac{\partial}{\partial t}\right) \tau(y, t)\right|_{y=0}=\left.\mu \frac{\partial u}{\partial y}\right|_{y=0}=\tau_{0} f(t), T(0, t)=T_{d}+\left(T_{w}-T_{d}\right) g_{1}(t), \\
u(d, t)=0, T(d, t)=T_{w} .
\end{gathered}
$$

with the following replacement for variable and parameters

$$
\begin{aligned}
& y^{*}=\frac{y}{d}, t^{*}=\frac{v t}{d^{2}}, u^{*}=\frac{d u}{v}, T^{*}=\frac{T-T_{d}}{T_{w}-T_{d}}, \lambda=\frac{\lambda_{1} v}{d^{2}}, \operatorname{Pr}=\frac{\mu c_{p}}{k}, \frac{1}{K}=\frac{\phi d^{2}}{k_{1}}, \\
& g\left(t^{*}\right)=g_{1}\left(\frac{d^{2}}{v} t^{*}\right), \tau_{1}=\frac{\tau_{0} d^{2}}{v \mu}, h\left(t^{*}\right)=f\left(\frac{d^{2}}{v} t^{*}\right), H_{a}=\sqrt{\frac{\sigma}{\mu}} B_{0} d, \tau^{*}=\frac{d^{2} \tau}{\mu \nu}, \\
& \mathrm{Gr}=\frac{g \beta\left(T_{w}-T_{d}\right) d^{3}}{v^{2}},
\end{aligned}
$$

and after dropping the $*$, dimensionless model is as

$$
\begin{gathered}
\frac{\partial^{2} u(y, t)}{\partial y^{2}}+G r\left(1+\lambda \frac{\partial}{\partial t}\right) T(y, t)-\left(1+\lambda \frac{\partial}{\partial t}\right) H_{a}^{2} u(y, t)-\frac{1}{K} u(y, t) \\
=\left(1+\lambda \frac{\partial}{\partial t}\right) \frac{\partial u(y, t)}{\partial t}, \\
\frac{\partial^{2} T(y, t)}{\partial y^{2}}=\operatorname{Pr} \frac{\partial T(y, t)}{\partial t},
\end{gathered}
$$

with dimensionless conditions

$$
\begin{gathered}
u(y, 0)=0=\frac{\partial u(y, 0)}{\partial t}, \tau(y, 0)=0, \\
\left.\left(1+\lambda \frac{\partial}{\partial t}\right) \tau(y, t)\right|_{y=0}=\left.\frac{\partial u}{\partial y}\right|_{y=0}=\tau_{1} h(t), T(0, t)=g(t), \\
u(1, t)=0, T(1, t)=1,
\end{gathered}
$$

where $u$ : is dimensionless fluid velocity, $T$ : dimensionless temperature for fluid, $T_{w}$ : temperature at wall, $\rho$ : density, $\mu$ :dynamic viscosity, $\lambda_{1}$ relaxation time, $v$ : kinematic viscosity, $k$ : thermal conductivity, $g$ : Gravitational acceleration, Pr: 
Prandtl number, $\tau_{0}$ : Constant shear stress, $k_{1}$ : permeability of medium, $B_{0}$ : magnetic field, $C_{p}$ : specific heat at constant pressure, $\phi$, porosity, $H_{a}$ : Hartmann number, $\beta$ : coefficient of volumetric expansion, and $\sigma$ : represented the electrical conduction for fluid.

\section{Solution of Problem}

As Equation (8) is not coupled with Equation (7), so we can solve separately Equation (8) for temperature field.

\subsection{Thermal Field}

Equation (8) from reduced to ordinary differential equation with the help of Laplace transform, as follows

$$
\frac{\partial^{2} \bar{T}(y, s)}{\partial y^{2}}=\operatorname{Pr} s T(y, s) .
$$

Transformed temperature $\bar{T}(y, s)$ satisfies the transformed condition $\bar{T}(0, s)=\bar{g}(s)$ and $\bar{T}(1, s)=\frac{1}{s}$ under all these conditions we obtain the following Laplace transform of temperature profile

$$
\bar{T}(y, s)=s \bar{g}(s)\left[\frac{\mathrm{e}^{(1-y) \sqrt{\operatorname{Pr} s}}-\mathrm{e}^{-(1-y) \sqrt{\operatorname{Pr} s}}}{2 s \sinh (\sqrt{\operatorname{Pr} s})}\right]+\frac{\mathrm{e}^{y \sqrt{\operatorname{Prs}}}-\mathrm{e}^{-y \sqrt{\operatorname{Pr} s}}}{2 s \sinh (\sqrt{\operatorname{Pr} s})} .
$$

Consider

$$
A_{1}(y, s)=\frac{\mathrm{e}^{(1-y) \sqrt{\operatorname{Pr} s}}-\mathrm{e}^{-(1-y) \sqrt{\operatorname{Pr} s}}}{2 s \sinh (\sqrt{\operatorname{Pr} s})}=\sum_{k=0}^{\infty}\left[\frac{\mathrm{e}^{-(2 k+y)} \sqrt{\operatorname{Pr} s}}{s}-\frac{\mathrm{e}^{-(2 k+2-y) \sqrt{\operatorname{Pr} s}}}{s}\right] .
$$

After the inversion Equation (14), we get

$$
a_{1}(y, t)=\sum_{k=0}^{\infty}\left[\operatorname{erfc}\left(\frac{(2 k+y) \sqrt{\operatorname{Pr}}}{2 \sqrt{t}}\right)-\operatorname{erfc}\left(\frac{(2 k+2-y) \sqrt{\operatorname{Pr}}}{2 \sqrt{t}}\right)\right] .
$$

Now by inversion of Laplace transform in Equation (13) we reach to the following result for temperature field in $t$-domain

$$
\begin{gathered}
T(y, t)=\left(L^{-1}\{s \bar{g}(s)\} * a_{1}(y, t)\right)+a_{2}(y, t), \\
T(y, t)=\dot{g}(t) * a_{1}(y, t)+a_{2}(y, t),
\end{gathered}
$$

where

$$
\begin{gathered}
a_{2}(y, t)=\sum_{k=0}^{\infty}\left[\operatorname{erfc}\left(\frac{(2 k+1-y) \sqrt{\operatorname{Pr}}}{2 \sqrt{t}}\right)-\operatorname{erfc}\left(\frac{(2 k+1+y) \sqrt{\operatorname{Pr}}}{2 \sqrt{t}}\right)\right], \\
T(y, t)=\int_{0}^{t} \dot{g}(t-\tau) \sum_{k=0}^{\infty}\left[\operatorname{erfc}\left(\frac{(2 k+y) \sqrt{\operatorname{Pr}}}{2 \sqrt{\tau}}\right)-\operatorname{erfc}\left(\frac{(2 k+2-y) \sqrt{\operatorname{Pr}}}{2 \sqrt{\tau}}\right)\right] \mathrm{d} \tau \\
+\sum_{k=0}^{\infty}\left[\operatorname{erfc}\left(\frac{(2 k+1-y) \sqrt{\operatorname{Pr}}}{2 \sqrt{t}}\right)-\operatorname{erfc}\left(\frac{(2 k+1+y) \sqrt{\operatorname{Pr}}}{2 \sqrt{t}}\right)\right] .
\end{gathered}
$$




\subsection{Calculation of Velocity}

Equation (7) reduces to ordinary equation by Laplace transform as follows

$$
\begin{gathered}
\frac{\partial^{2} \bar{u}(y, s)}{\partial y^{2}}-\left[s(1+\lambda s)+H_{a}^{2}(1+\lambda s)+\frac{1}{K}\right] \bar{u}(y, s) \\
=-\frac{\operatorname{Gr}(1+\lambda s) \bar{g}(s)}{\sinh (\sqrt{\operatorname{Pr} s})} \sinh ((1-y) \sqrt{\operatorname{Pr} s})-\frac{\operatorname{Gr}(1+\lambda s)}{s \sinh (\sqrt{\operatorname{Pr} s})} \sinh (y \sqrt{\operatorname{Pr} s}) \\
\frac{\partial^{2} \bar{u}(y, s)}{\partial y^{2}}-B_{0}(s) \bar{u}(y, s)=-B_{1}(s) \sinh ((1-y) \sqrt{\operatorname{Pr} s})-B_{2}(s) \sinh (y \sqrt{\operatorname{Pr} s}) .
\end{gathered}
$$

Equation (21) satisfies the conditions, $\frac{\partial \bar{u}(y, s)}{\partial y}=\tau_{1} \bar{h}(s)$, and $\bar{u}(1, s)=0$. Using these conditions we can obtain the following Laplace transform of velocity field

$$
\begin{aligned}
\bar{u}(y, s)= & \frac{B_{4}(s) \sqrt{B_{0}(s)}+B_{3}(s) \mathrm{e}^{-\sqrt{B_{0}(s)}}}{\sqrt{B_{0}(s)}\left(\mathrm{e}^{\sqrt{B_{0}(s)}}+\mathrm{e}^{-\sqrt{B_{0}(s)}}\right)} \exp \left(y \sqrt{B_{0}(s)}\right) \\
& +\frac{B_{4}(s) \sqrt{B_{0}(s)}-B_{3}(s) \mathrm{e}^{\sqrt{B_{0}(s)}}}{\sqrt{B_{0}(s)}\left(\mathrm{e}^{\sqrt{B_{0}(s)}}+\mathrm{e}^{-\sqrt{B_{0}(s)}}\right)} \exp \left(-y \sqrt{B_{0}(s)}\right) \\
& +\frac{B_{1}(s)}{B_{0}(s)-\operatorname{Pr} s} \sinh ((1-y) \sqrt{\operatorname{Pr} s})+\frac{B_{2}(s)}{B_{0}(s)-\operatorname{Pr} s} \sinh (y \sqrt{\operatorname{Pr} s}),
\end{aligned}
$$

where

$$
\begin{gathered}
B_{0}(s)=s(1+\lambda s)+(1+\lambda s) H_{a}^{2}+\frac{1}{K}, \\
B_{1}(s)=\frac{G r(1+\lambda s) \bar{g}(s)}{\sinh (\sqrt{\operatorname{Pr} s})}, \\
B_{2}(s)=\frac{G r(1+\lambda s)}{s \sinh (\sqrt{\operatorname{Pr} s})}, \\
B_{3}(s)=\tau_{1} \bar{h}(s)+\frac{B_{1}(s) \sqrt{\operatorname{Pr} s} \cosh (\sqrt{\operatorname{Pr} s})-\frac{B_{2}(s) \sqrt{\operatorname{Pr} s}}{B_{0}(s)-\operatorname{Pr} s},-\operatorname{Pr} s}{B_{4}(s)=-\frac{B_{2}(s)}{B_{0}(s)-\operatorname{Pr} s} \sinh (\sqrt{\operatorname{Pr} s}) .}
\end{gathered}
$$

Equation (22) is very complicated and inverse Laplace transform is not possible by ordinary inversion formulas. Therefore, we will use numerical techniques namely Stehfest's and Tzou's algorithms for inversion of Laplace transform in Equation (22).

\subsection{Graphical Results and Discussions}

Maxwell fluid flowing through poriferous medium under the influence of a constant magnetic has been studied in this article. Prescribed Maxwell fluid model solved by integral transform method and find an analytical. Also results for ve- 
locity and temperature are presented by some graphics to illustrate the effect of parameters. All the graphs are plotted for functions $g(t)=1-\mathrm{e}^{-t}$ and $h(t)=1-\cos t$. The inversion is made by performing the Stehfest's algorithm and Tzou's algorithms to make a validation for our obtained results.

Figure 1 depicts the temperature dependence on Prandtl number while fixing values of other involved parameters. It is clear by the graphic that by increasing value Pr the thermal profile fall. An increasing Pr meant that we trimming down the heat conduction and improving the viscosity. Figure 2 is sketched to study the influence of time $t$ on the thermal profile of fluid. By graphic illustration it is clear that for progressive time $t$ the temperature profile fall down.

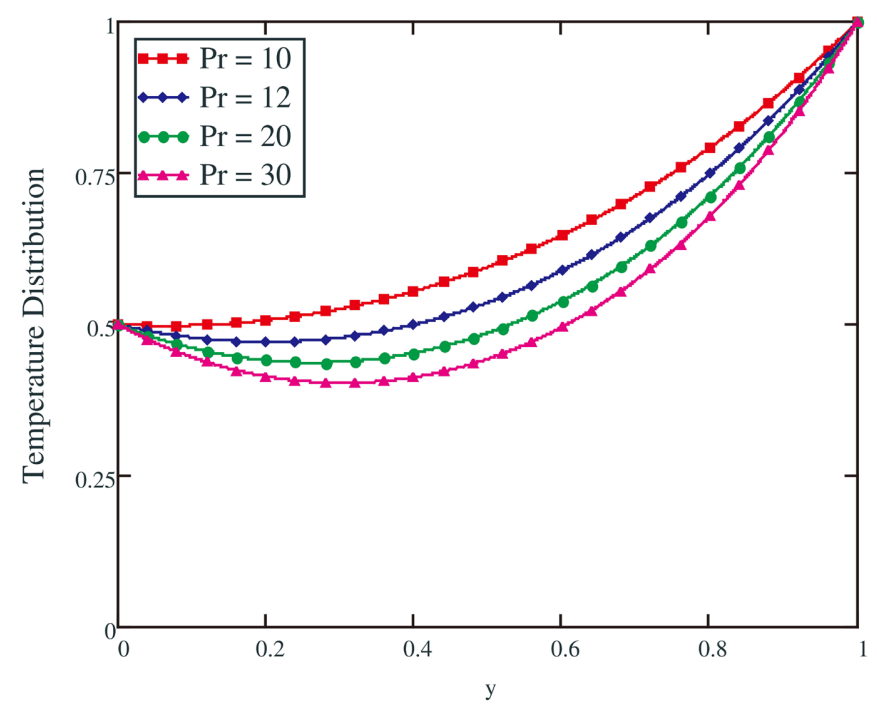

Figure 1. Temperature profiles against $y$ due to $\operatorname{Prgiven}$ by the Equation (19).

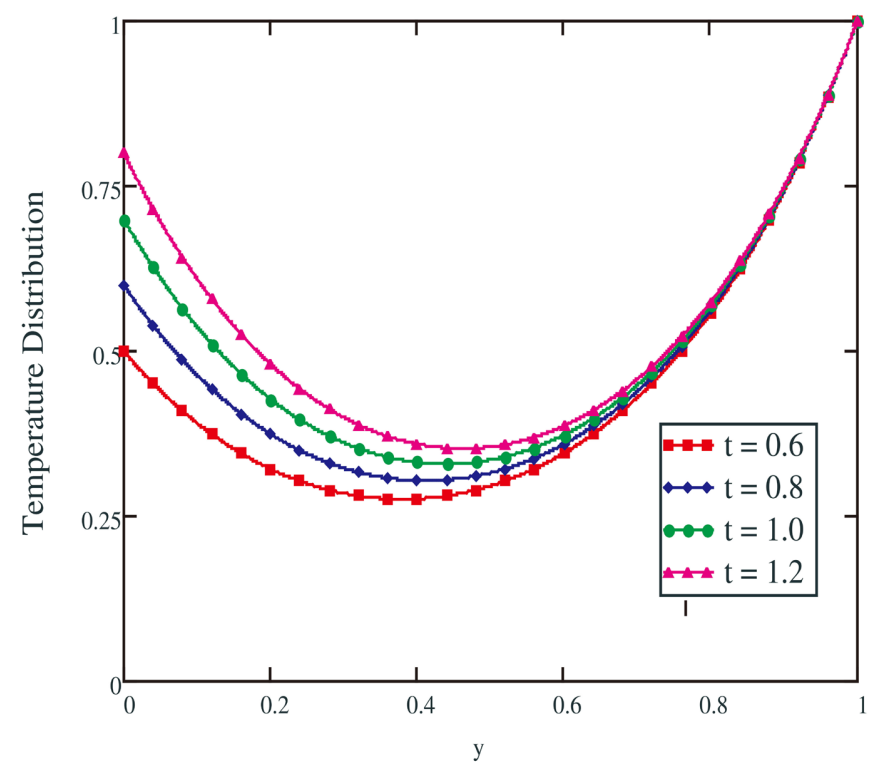

Figure 2. Temperature profiles against $y$ due to $t$ given by the Equation (19). 
Figure 3 is drawn to elaborated subjectivity of Grashof number Gr over the fluid's motion. For every successive increment to the value of $G r$ the fluid speed up and this is because the fact that an increasing $G r$ that buoyancy forces increases, causing an increase in the fluid's motion. Figure 4 is sketch to see the effect of Prandtl number on fluid's velocity and it is noted that fluid slow down with the increasing Pr.

Figure 5 is drawn to discuss the effect of porosity $K$ and from the profile pattern it is observed that developing value of $K$ the fluid's velocity slow down. Physically, this result can be achieved when the voids of the permeable medium are very small so that such medium may offer considerable opposition to flow. In Figure 6, the influence of Hartmann number $H_{\alpha}$ has been explained. An

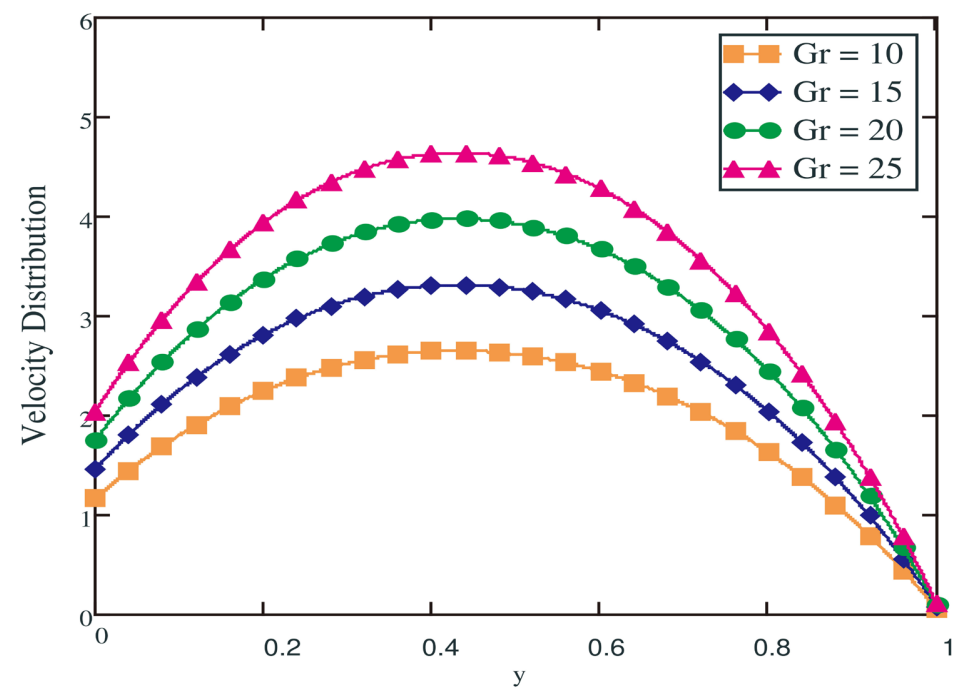

Figure 3. Velocity profiles against $y$ due to Grashof number $G r$ given by the Equation (22).

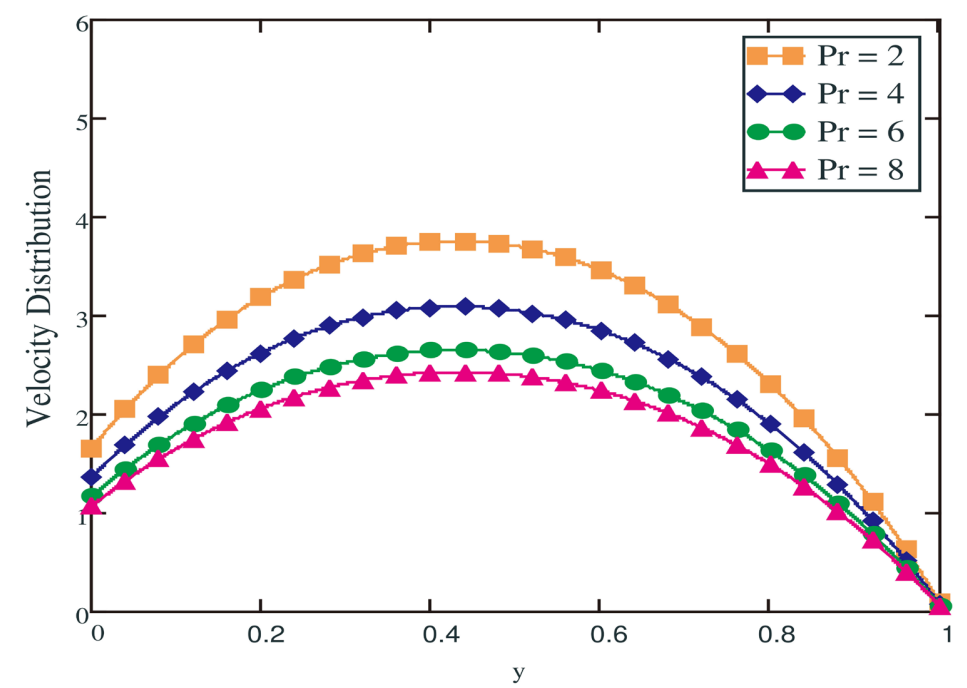

Figure 4. Velocity profiles against $y$ due to Prandlt number $P r$ given by the Equation (22). 


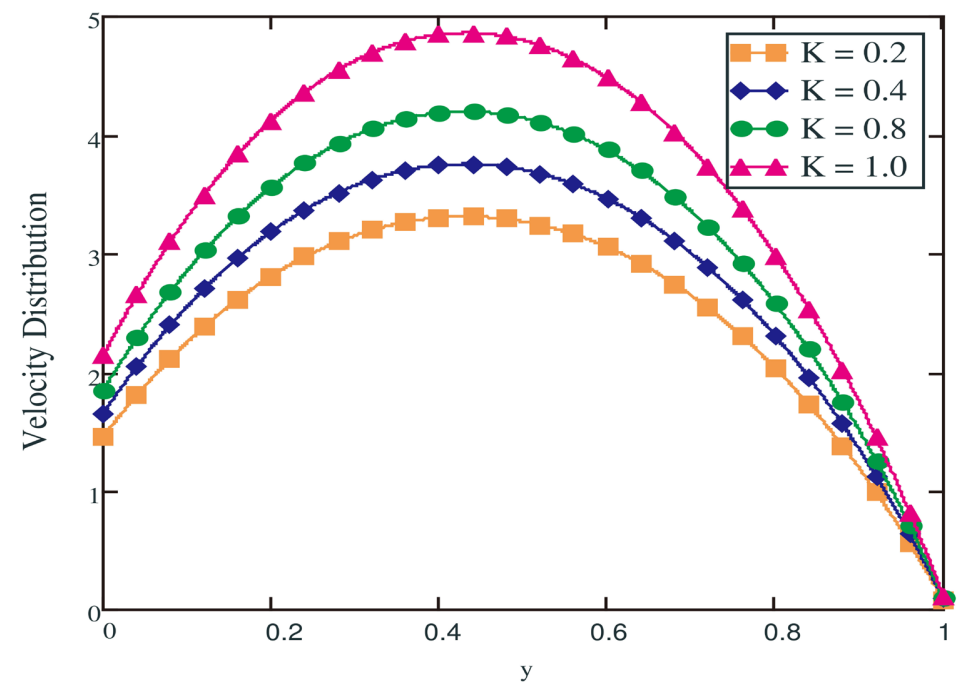

Figure 5 . Velocity profiles due to porocity parameter $K$ given by the Equation (22).

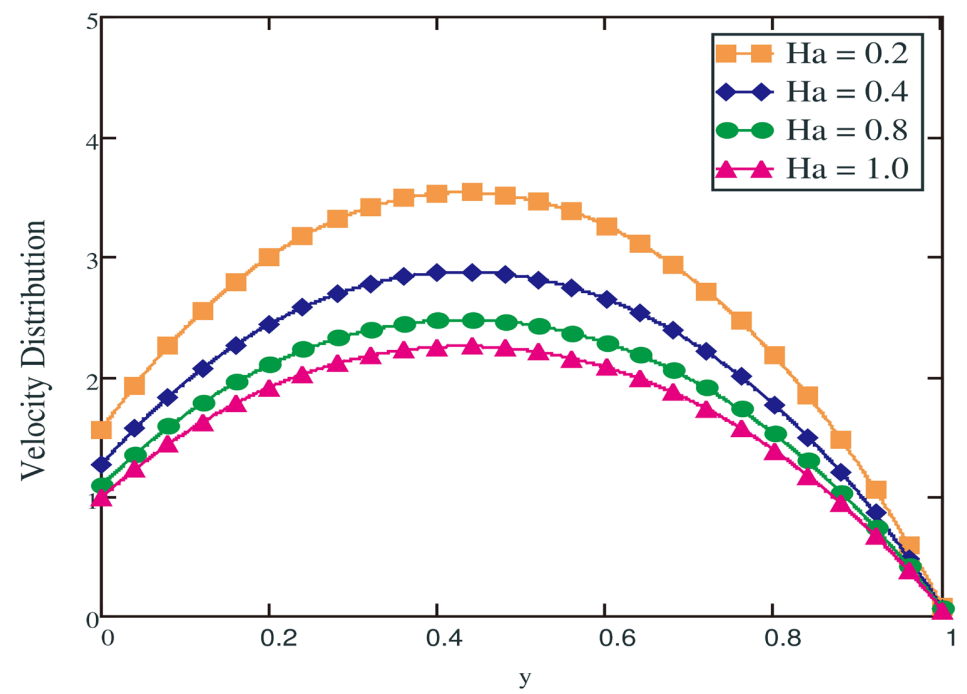

Figure 6. Velocity profiles due to Hatmann number $H_{a}$ given by the Equation (22).

enhancing $t$ value of Hartmann number decreases the velocity of the fluid but gave an increment in the thickness of the boundary layer. The reason behind that the Lorentz force resisted fluid motion more vigorously for larger values of magnetic field parameter and hence fluid tend to slow down. Figure 7 illustrated the influence of $\lambda$. For increasing value of $\lambda$, the motion of fluid and boundary layer thickness increases. Figure 8 drawn to see the objectivity of fluid's velocity over the time and it is noted that for delaying the instants the fluid's motion as well as the boundary layer thickness boosted. Figure 9 and Figure 10 are drawn to present the inversion results for the Laplace transform in Equation (22) that are obtained by Stehfestís and Tzouís algorithms and overlapping profiles is an evidence of validity of inversion results for velocity. 


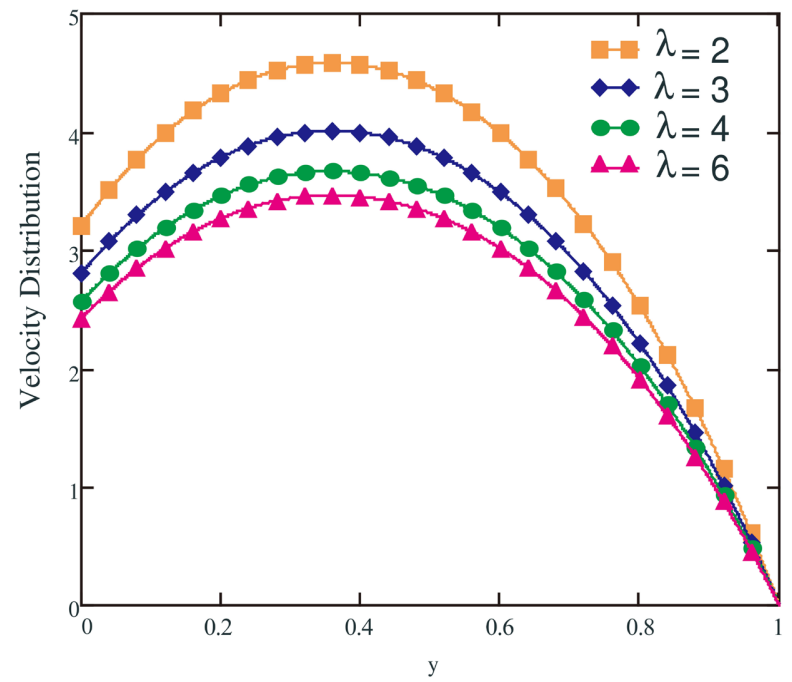

Figure 7. Velocity profile due to relaxation time $\lambda$ given by the Equation (22).

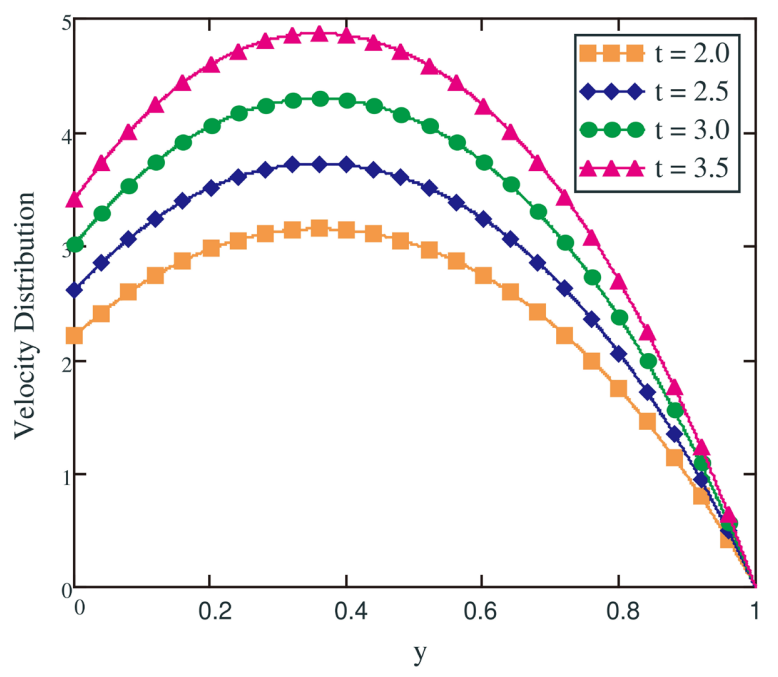

Figure 8. Velocity profiles due to time $t$ given by the Equation (22).

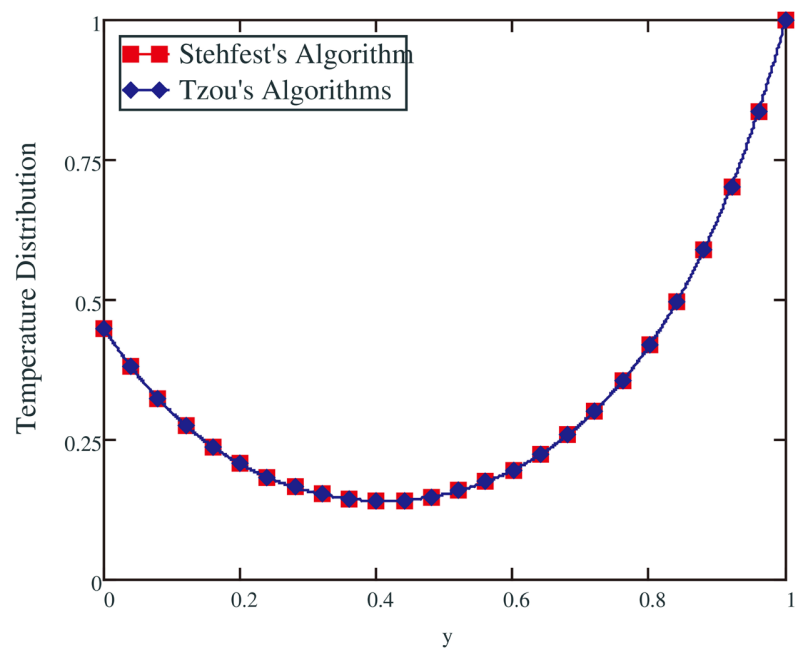

Figure 9. Temperature $T(y, t)$ profiles for Equation (19). 


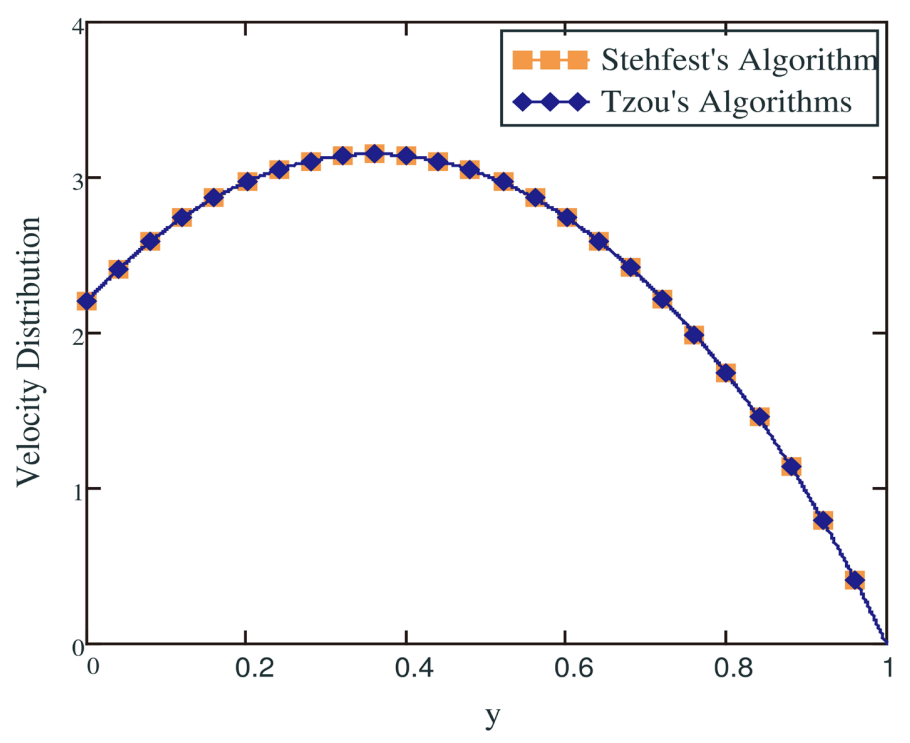

Figure 10. Velocity $u(y, t)$ profiles against y for the Equation (22).

\section{Conclusions}

This paper is design for Maxwell fluid flow model and is solved to establish the analytical expressions for temperature and velocity with the help of Laplace transform method. Obtained results for Velocity and temperature are plotted to see the influence of parameters graphically. Inversion of transformed temperature and velocity fields are made by Tzouís and Stehfestís algorithms and inverted results are presented in Figure 9 and Figure 10. Some important remarkable conclusions of present study are outlined as follows:

1) An enhancing value of $\operatorname{Pr}$ falls down temperature.

2) Temperature field increases for time development.

3) Velocity field increases for $G r$ and $\lambda$.

4) Velocity slows down for $\operatorname{Pr}, K$ and $H_{a}$.

5) Achieved profiles for velocity and temperature through both inversion algorithms namely, Stehfestís and Tzouís are equivalent.

\section{Conflicts of Interest}

The authors declare no conflicts of interest regarding the publication of this paper.

\section{References}

[1] Merkin, J.H. (1994) Natural Convection Boundary Layer Plow on a Vertical Surface with Newtonian Heating. International Journal of Heat and Fluid Flow, 15, 392-398. https://doi.org/10.1016/0142-727X(94)90053-1

[2] Hayat, T. and Abbas, Z. (2008) Heat Transfer Analysis on the MHD Flow of a Second Grade Fluid in a Channel with Porous Medium. Chaos, Solitons and Fractals, 38, 556-567. https://doi.org/10.1016/j.chaos.2006.12.004

[3] Zhao, J., Zheng, L., Zhang, X. and Liu, F. (2016) Convection Heat and Mass Trans- 
fer of Fractional MHD Maxwell Fluid in Porous Medium with Soret and Dufour Effects. International Journal of Heat and Mass Transfer, 103, 203-210. https://doi.org/10.1016/j.ijheatmasstransfer.2016.07.057

[4] Arifuzzaman, S.M., Khan, M.S., Islam, M.S., Islam, M.M., Rana, B.M.J., Biswas, P. and Ahmmed, S.F. (2017) MHD Maxwell Fluid Flow in Presence of Nano-Particle through a Vertical Porous Plate with Heat-Generation, Radiation Absorption and Chemical Reaction. Frontier in Heat and Mass Transfer, 9, 1-14.

https://doi.org/10.5098/hmt.9.25

[5] Jamil, M. and Abro, K.L. (2015) MHD Maxwell Fluid with Non Linear Velocity over the Boundary. ICEC.

[6] Mahsud, Y., Shah, N.A. and Vieru, D. (2017) Influence of Time-Fraction Derivatives on the Boundary Layer Flow of Maxwell Fluid. Chinese Journal of Physics, 55, 1340-1351. https://doi.org/10.1016/j.cjph.2017.07.006

[7] Riaz, M.B., Imran, M.A. and Shabir, K. (2016) Analytic Solutions of Oldroyd-B Fluid with Fractional Derivatives in a Circular Duct That Applies a Constant Couple. Alexandria Engineering Journal, 55, 3267-3275.

[8] Samiulhaq, A.S., Vieru, D., Khan, I. and Shafie, S. (2014) Unsteady Magnetohydrodynamic Free Convection Flow of a Second Grade Fluid in a Porous Medium with Ramped Wall Temperature. PLOS ONE, 9, 1-9.

[9] Kaushik, P., Mandal, S. and Chakraborty, S. (2017) Transient Electroosmosis of a Maxwell Fluid in a Rotating Microchannel. Electrophoresis, 38, 2741-2748. https://doi.org/10.1002/elps.201700090

[10] Fong, C.M., De Kee, D. and Marcos, B. (1993) Transient Flow of a Maxwell Fluid on Rotating Disk. Journal of Applied Physics, 74, 40. https://doi.org/10.1063/1.354125

[11] Hayat, T., Hina, S. and Hendi, A.A. (2012) Slip Effects on Peristaltic Transport of a Maxwell Fluid with Heat and Mass Transfer. Journal of Mechanics in Medicine and Biology, 12, Article ID: 1250001. https://doi.org/10.1142/S0219519412004375

[12] Rosti, M.E., Cortelezzi, L. and Quadrio, M. (2015) Direct Numerical Simulation of Turbulent Channel Flow over Porous Walls. Journal of Fluid Mechanics, 784, 396-442. https://doi.org/10.1017/jfm.2015.566

[13] Nadeem, S., Rehman, A., Lee, C. and Lee, J. (2012) Boundary Layer Flow of Second Grade Fluid in a Cylinder with Heat Transfer. Mathematical Problems in Engineering, 2012, Article ID: 640289. https://doi.org/10.1155/2012/640289

[14] Middleman, S. (1998) An Introduction Fluid Dynamics. John Wiley and Sons, Inc., Hoboken.

[15] Akhtar, S. and Shah, N.A. (2016) Exact Solution for Some Unsteady Flow of a Couple Stress Fluid between Parallel Plates. Ain Shams Engineering Journal, 9, 985-992.

[16] Escandon, J., Jimnesez, E., Hernandez, C., Bautsta, O. and Mendez, F. (2015) Transient Electroosmotic Flow of Maxwell Fluids in a Slit Microchannel with Asymmetric Zeta Potentials. European Journal of Mechanics B/ Fluids, 53, 180-189. https://doi.org/10.1016/j.euromechflu.2015.05.001

[17] Fetecau, C. and Vieru, D. (2007) On Some Helical Flows of Oldroyd-B Fluids. Acta Mechanica, 189, 53-63. https://doi.org/10.1007/s00707-006-0407-7

[18] Imran, M., Athar, M. and Kamran, M. (2011) On the Unsteady Rotational Flow of a Generalized Maxwell Fluid through a Circular Cylinder. Archive of Applied Mechanic, 81, 1659-1666. https://doi.org/10.1007/s00419-011-0509-0

[19] Fox, R.W. and Macdonald, A. (2008) Introduction to Fluid Mechanics. 5th Edition, 
School of Mechanical Engineering, Purdue University, West Lafayette.

[20] Vieru, D., Akhtar, W. and Fetecau, C. (2007) Starting Solutions for the Oscillating Motion of a Maxwell Fluid in Cylindrical Domains. Meccanica, 42, 573-583.

https://doi.org/10.1007/s11012-007-9081-7

[21] Vieru, D., Fetecau, C. and Fetecau, C. (2015) Time-Fractional Free Convection Flow near a Vertical Plate with Newtonian Heating and Mass Diffusion. Thermal Science, 19, 85-98. https://doi.org/10.2298/TSCI15S1S85V

[22] Imran, M.A., Khan, I., Ahmad, M., Shah, N.A. and Nazar, M. (2017) Heat and Mass Transport of Differential Type Fluid with Non-Integer Order Time-Fractional Caputo Derivatives. Journal of Molecular Liquids, 229, 67-75. https://doi.org/10.1016/j.molliq.2016.11.095

[23] Bose, D. and Basu, U. (2013) Unsteady Incompressible Viscoelastic Flow of a Generalised Maxwell Fluid between Two Rotating Infinite Parallel Coaxial Circular Disks. Open Journal of Fluid Dynamics, 3, 57-63. https://doi.org/10.4236/ojfd.2013.32008

[24] Nazar, M., Athar, M. and Akhtar, W. (2008) Axial Couette Flow of Second Grade Fluid Due to a Longitudinal Time Dependent Shear Stress. Proceedings of 10 th International Conference Zaragoza-Pau on Applied Mathematics and Statistics, Jaca, 13-19.

[25] Sultan, Q., Nazar, M., Ali, U. and Imran, M. (2013) Unsteady Flow of Oldroyd-B Fluid through Porous Rectangular Duct. International Journal of Nonlinear Science, 15, 195-211.

[26] Sultan, Q., Nazar, M., Imran, M. and Ali, U. (2014) Flow of Generalized Burgers Fluid between Parallel Walls Induced by Rectified Sine Pulses Stress. Boundary Value Problems, 2014, Article No. 152. https://doi.org/10.1186/s13661-014-0152-0

[27] Khan, I., Ahmad, M., Shah, N.A. and Imran, M.A. (2017) Effects of Non-Integer Order Time Fractional Derivative on Coupled Heat and Mass Transfer of MHD Viscous Fluid over an Infinite Inclined Plane with Heat Absorption. International Journal of Innovative Research in Science, Engineering and Technology, 6, 1-11.

[28] Riaz, M.B., Imran, M.A. and Shabbir, K. (2016) New Exact Solutions for the Flow of Generalized Maxwell Fluid. Journal of Computational and Theoretical Nanoscience, 13, 5254-5257. https://doi.org/10.1166/jctn.2016.5409

[29] Akhtar, W. and Nazar, M. (2008) Exact Solutions for the Rotational Flow of Generalized Maxwell Fluids in a Circular Cylinder. Bulletin mathematique de la Societe des Sciences Mathematiques de Roumanie, 51, 93-101. 